\title{
ANÁLISE DE COMPONENTES PRINCIPAIS APLICADA À CIÊNCIA DE ALIMENTOS: ESTUDO DE CASO COM PEQUI
}

\author{
Aline Inacio Alves ${ }^{1}$ \\ Marcela Zonta Rodrigues ${ }^{2}$ \\ Ellen Silva Lago Vanzela ${ }^{3}$ \\ Paulo Cesar Stringheta ${ }^{4}$ \\ Afonso Mota Ramos ${ }^{5}$
}

Resumo: Com este trabalho se objetivou avaliar a potencialidade da análise de componentes principais para a interpretação de resultados de um experimento com microencapsulamento de pequi. O experimento foi realizado no laboratório de Tecnologia de Frutas e Hortaliças do Departamento de Engenharia de Alimentos da Universidade Federal de Viçosa. As variáveis utilizadas neste trabalho foram extraídas de um estudo físico-químico e morfológico obtidos a partir de extrato de pequi. A análise de componentes foi realizada com base na matriz de correlação existente entre as componentes e as variáveis reais transformadas, de forma a identificar novas variáveis que explicam a maior parte da variabilidade, gerandose novos valores para cada parcela experimental correspondente às componentes principais. Para cada temperatura utilizada no trabalho foram ajustadas apenas duas componentes capazes de explicar mais de 90\% da variância dos dados originais. A análise de componentes principais foi eficiente neste estudo, gerando componentes interpretáveis reduzindo a dimensionalidade do problema se a perda de informações. Palavras-chave: Estatística multivariada; Engenharia de alimentos; PCA.

\footnotetext{
1 Universidade Federal de Viçosa, Brasil. E-mail: aline_inacio27@hotmail.com.

2 Universidade Federal de Viçosa, Brasil. E-mail: marcela_vrb@yahoo.com.br.

3 Universidade Estadual Paulista, Brasil. E-mail: ellenlagovanzela@hotmail.com.

4 Universidade Federal de Viçosa, Brasil. E-mail: pstringheta@gmail.com.

5 Universidade Federal de Viçosa, Brasil. E-mail: amramos@ufv.br.
} 